Bundesgesundheitsbl 2020 - 63:1250-1261 https://doi.org/10.1007/s00103-020-03216-6 Eingegangen: 29. November 2019

Angenommen: 21. August 2020

Online publiziert: 24. September 2020

(c) Der/die Autor(en) 2020

Diana Kurch-Bek' $\cdot$ Christian Gallowitz' ${ }^{1}$ Axel Baumgarten ${ }^{2}$ Bernhard Tenckhoff' $~$ Matthias an der Heiden ${ }^{3}$

'Innovation, strategische Analyse und IT-Beratung, Kassenärztliche Bundesvereinigung, Berlin, Deutschland

${ }^{2}$ Deutsche Arbeitsgemeinschaft niedergelassener Ärzte in der Versorgung HIV-Infizierter (dagnä) e. V., Berlin, Deutschland

${ }^{3}$ Fachgebiet für HIV/AIDS und andere sexuell oder durch Blut übertragbare Infektionen, Abteilung für Infektionsepidemiologie, Robert Koch-Institut, Berlin, Deutschland

\title{
Häufigkeiten von Patienten mit HIV in der ambulanten Versorgung in Deutschland - Eine Analyse auf Basis von Sekundärdaten
}

\section{Hintergrund und Fragestellung}

Im Jahr 2016 lebten nach Angaben des Gemeinsamen Programms der Vereinten Nationen für HIV/Aids (UNAIDS) weltweit ca. 36,7 Mio. Menschen mit dem humanen Immundefizienzvirus (HIV), davon erwarben ca. 1,8 Mio. Menschen eine HIV-Neuinfektion im Jahr 2016. Das entspricht etwa 5000 Neuinfektionen pro Tag weltweit. Frauen und Männer sind international in gleichem Maße betroffen. $64 \%$ der Menschen mit diagnostiziertem HIV leben südlich der Sahara (Subsahara-Afrika; [1]).

In Deutschland lebten im Jahr 2017 nach Berechnungen des Robert KochInstituts (RKI) ca. 86.000 Menschen mit einer HIV-Infektion, von denen sich etwa 2600 Menschen neu mit HIV infiziert hatten. Von den HIV-Erstdiagnosen betrafen $80 \%$ Männer und $20 \%$ Frauen. Vorrangig ist die Geschlechterverteilung in Deutschland durch die hohe Betroffenheit von Männern, die Sex mit Männern haben (MSM; 65\%), geprägt. Über einen heterosexuellen Kontakt hingegen hatten sich etwa $25 \%$ infiziert. Ungefähr $5 \%$ der Neudiagnosen waren auf die Übertragung von HIV durch intravenösen Drogengebrauch (IVD) zurückzuführen. 73.100 Infektionen wurden im In- land oder von Menschen deutscher Herkunft im Ausland erworben und verteilen sich zu etwa $73 \%$ auf MSM und zu etwa $15 \%$ auf Infektionen über heterosexuelle Kontakte. Rund $11 \%$ dieser Infektionen wurden über den Konsum IVD erworben. Jeweils geringer als $1 \%$ war der Anteil der Patienten, die sich über Bluttransfusionen größtenteils in den 1980erJahren in Deutschland oder über ihre Mutter, deren Herkunft meist außerhalb von Deutschland liegt, mit HIV infiziert hatten [2-4].

Eine frühe Diagnose einer HIV-Infektion ist für die Patienten und die Gesellschaft von hoher Bedeutung, um den Zugang zur antiretroviralen Therapie zu ermöglichen und damit effektiv weitere Transmissionen zu vermeiden. In Deutschland besteht ein flächendeckendes Testangebot, dennoch wird ein Teil der HIV-Infektionen erst in einem späten Stadium erkannt [3]. Trotz der guten Therapiemöglichkeiten behält der HIVTest in der ärztlichen Praxis eine Sonderstellung. Ein Patient muss aufgrund medizinischer, sozialer und rechtlicher Folgen einem HIV-Test vorher ausdrücklich zustimmen. In einigen europäischen Ländern, wie beispielsweise Frankreich, gehören das Testangebot und die Beratung im Rahmen der hausärztlichen
Versorgung zum Standard. Darüber hinaus sind Einsendetests zugelassen, welche einen niedrigschwelligen Zugang zum Testangebot darstellen [5-7]. Eine HIV-Infektion ist eine meldepflichtige Krankheit gemäß $₫ 7$ Abs. 3 Infektionsschutzgesetz (IfSG), welche durch das Labor nichtnamentlich, jedoch mit einer fallbezogenen Verschlüsselung über spezielle Meldebögen direkt an das RKI übermittelt wird. Diese Daten stellen ein wichtiges Instrument zur epidemiologischen Beurteilung der HIV-Epidemie dar.

Das RKI veröffentlicht jährlich Zahlen $\mathrm{zu}$ HIV-Neuinfektionen und der Gesamtzahl von Menschen mit HIV in Deutschland. Hierzu werden verschiedene Datenquellen der Routine-Surveillance genutzt, wie Daten der Laborberichtsverordnung, Meldedaten nach dem IfSG, Aidsfallberichte, HIV/Aids-Todesfallberichte, die Todesursachenstatistik des Bundes sowie bundesweite Daten des Informationsdienstleisters Insight Health zur Verordnung von antiretroviralen Medikamenten bei gesetzlich versicherten Patienten [2-4]. Im internationalen Kontext wurden mehrere Methoden entwickelt, um anhand von zur Verfügung stehenden Daten (z.B. klinischen Registern, Meldedaten nach 


\begin{tabular}{|c|c|c|c|}
\hline \multicolumn{2}{|c|}{ Operationalisierungskriterien } & GOP/ICD-Code & Beschreibung \\
\hline \multirow[t]{4}{*}{ Test } & \multirow[t]{3}{*}{ Antikörpersuchtests } & 01811 & HIV-Immunoassay (Mutterschaftsvorsorge) \\
\hline & & 32575 & HIV-1- oder HIV-1/2-Antikörper - Immunassay \\
\hline & & 32576 & HIV-2-Antikörper - Immunoassay \\
\hline & Absicherungstest & 32660 & HIV-1-, HIV-2-Antikörper Western Blot \\
\hline \multirow{3}{*}{\multicolumn{2}{|c|}{ HIV-Betreuungsleistung }} & 30920 & Zusatzpauschale für die Behandlung von HIV-Infizierten \\
\hline & & 30922 & Zuschlag I zur GOP 30920 Behandlung von HIV-Infizierten \\
\hline & & 30924 & Zuschlag II zur GOP 30920 Behandlung von HIV-Infizierten \\
\hline \multirow{5}{*}{\multicolumn{2}{|c|}{ HIV-Diagnose }} & B20-B24 & HIV-Krankheit \\
\hline & & 098.7 & HIV-Krankheit, die Schwangerschaft, Geburt und Wochenbett kompliziert \\
\hline & & U60-U61 & Stadieneinteilung der HIV-Infektion \\
\hline & & U85 & HIV mit Resistenz gegen Virostatika oder Proteinaseinhibitoren \\
\hline & & $\mathrm{Z21}$ & Asymptomatische HIV-Infektion \\
\hline
\end{tabular}

IfSG) die Inzidenz und Prävalenz von HIV zu berechnen [8-12].

Bisher gibt es nur vereinzelte Studien zur bundesweiten Abschätzung der administrativen Prävalenz und Inzidenz von Menschen mit HIV auf der Basis von Sekundärdaten [13-15]. Im Rahmen der Untersuchung stützen wir uns auf HIVDiagnosen und HIV-spezifische Leistungen, welche in der ambulanten Versorgung in Anspruch genommen werden können [16].

In den untersuchten Leistungsdaten werden Patienten mit gesetzlicher Krankenversicherung (GKV), die erstmals eine HIV-Diagnose und/oder HIV-spezifische Leistungen in der ambulanten Versorgung im Betrachtungszeitraum erhalten haben, nach den Operationalisierungskriterien (gemäß • Tab. 1) als „erstmals ambulant versorgte Patienten mit HIV“ bezeichnet. Hierbei handelt es sich nicht um eine "administrative“ Inzidenz, da die Patienten die erstmalige Diagnosestellung oder Feststellung einer HIV-Infektion in einem unbestimmten Zeitraum vor der Leistungsinanspruchnahme erhalten haben können. Die verwendeten Sekundärdaten können keine Auskunft zu Patienten geben, bei denen außerhalb der ambulanten Versorgung eine HIV-Infektion diagnostiziert wurde und die ggf. anschließend keine oder eine spezialisierte Versorgung in Anspruch genommen haben.Zur Untersuchung der bundesweiten administrativen Prävalenz werden GKV-Patienten mit einer HIVDiagnose und/oder HIV-spezifischen
Leistungen, die ambulante Versorgung (kurz: ambulant versorgte Patienten mit $H I V)$ in Anspruch genommen haben, nach den Operationalisierungskriterien (gemäß • Tab. 1) betrachtet.

Die vorliegende Studie bestimmt die bundesweite Anzahl der ambulant versorgten Patienten mit HIV und der erstmals ambulant versorgten Patienten mit HIV populationsbasiert für alle gesetzlich krankenversicherten Patienten in Deutschland anhand von Sekundärdaten. Folgende Fragestellungen werden für die einzelnen Jahreskohorten untersucht:

- Wie hoch ist die Anzahl der erstmals ambulant versorgten Patienten mit HIV?

- Wie hoch ist die Anzahl der ambulant versorgten Patienten mit HIV?

- Wie viele Patienten nehmen in der ambulanten Versorgung einen HIVTest in Anspruch?

Ferner wird ein kurzer Exkurs zu den jährlichen Veröffentlichungen des RKI zu HIV und den hier präsentierten Sekundärdaten unternommen.

\section{Methodik}

\section{Datengrundlage}

Datengrundlage sind die bundesweiten ambulanten, kollektivvertraglichen Abrechnungsdaten gemäß $₫ 295$ und $\$ 87 c$ Sozialgesetzbuch (SGB) V der Jahre 2009 bis 2018 (Beobachtungszeitraum).
Enthalten sind alle gesetzlich Krankenversicherten mit mindestens einem Arztkontakt im kollektivvertraglichen Bereich (kurz: ambulante Versorgung) im entsprechenden Abrechnungszeitraum. Darüber hinaus sind Informationen über abgerechnete Diagnosen gemäß ICD-10 GM (International Statistical Classification of Diseases and Related Health Conditions, 10. Revision, German Modification) und Leistungen in Form von Gebührenordnungspositionen (GOPen) auf Basis des Einheitlichen Bewertungsmaßstabs (EBM) enthalten $[17,18]$. Es wurden ausschließlich gesicherte Diagnosen einbezogen, um Dokumentationseffekte auszuschließen, „Verdacht-auf“-, „Ausschluss-von“- und „Zustand-nach“-Diagnosen blieben unberücksichtigt.

In den Daten werden die Testung, die Leistungen zur HIV-Betreuung sowie die HIV-Diagnose von Patienten mittels der GOP sowie der gesicherten Diagnosen operationalisiert. In den Analysen wurde das Aufgreifkriterium des Bundesversicherungsamtes (BVA) „,in mindestens zwei unterschiedlichen Quartalen (M2Q)“verwendet [19]. • Tab. 1 gibt einen Überblick über die GOP und ICD10-Codes, die für die jeweilige Operationalisierung angewendet wurden.

\section{Bildung der Patienten- und Studienkohorten}

Für die vorliegende Untersuchung wurden Patientenkohorten jeweils für die 
Bundesgesundheitsbl 2020 -63:1250-1261 https://doi.org/10.1007/s00103-020-03216-6

(c) Der/die Autor(en) 2020

D. Kurch-Bek · C. Gallowitz · A. Baumgarten · B. Tenckhoff · M. an der Heiden

\section{Häufigkeiten von Patienten mit HIV in der ambulanten Versorgung in Deutschland - Eine Analyse auf Basis von Sekundärdaten}

\section{Zusammenfassung}

Hintergrund. Jährlich veröffentlicht das Robert Koch-Institut (RKI) die Zahl der HIVErstdiagnosen und die Gesamtzahl von Menschen mit HIV-Diagnosen in Deutschland. Eine Bestimmung mithilfe von Sekundärdaten erfolgte bislang nur mit Arzneimitteldaten.

Ziel der Arbeit. Auf Basis von Leistungsdaten wird die Anzahl von erstmals ambulant versorgten HIV-Patienten unter gesetzlich Krankenversicherten bestimmt sowie die Gesamtzahl von GKV-Patienten mit HIV in der ambulanten Versorgung. HIV-Testraten für Deutschland werden untersucht.

Material und Methoden. Die Leistungsdaten der gesetzlich Krankenversicherten aus dem ambulanten kollektivvertraglichen Sektor (kurz: ambulante Versorgung) liegen für den
Beobachtungszeitraum 2009 bis 2018 vor. Für die Jahre 2011 bis 2017 wurden Jahreskohorten auf Basis von Patientenentitäten gebildet und hinsichtlich der HIV-Diagnose, der HIVspezifischen Betreuungsleistungen und HIVTestraten untersucht.

Ergebnisse. Jährlich werden etwa $6000 \mathrm{~Pa}-$ tienten mit HIV erstmals ambulant versorgt. Die Gesamtzahl der ambulant versorgten Patienten mit HIV betrug im Jahr 2011 etwa $59.300(0,106 \%)$ und stieg zum Jahr 2017 auf etwa $80.800(0,141 \%)$ an. Der durchschnittliche Anstieg der Gesamtzahl um etwa 3600 Patienten pro Jahr lag damit deutlich unter der geschätzten Zahl jährlich neu versorgter Patienten.
Schlussfolgerung. Die Ergebnisse können erste Anhaltspunkte zur ambulanten Versorgung von Patienten mit HIV geben. Die vorliegenden Sekundärdaten geben die Möglichkeit, eine weitere epidemiologische Datenquelle zur bevölkerungsbezogenen Darstellung der administrativen Prävalenz von HIV zu erschließen. Es besteht Forschungsbedarf zu Patienten, welche erstmals ambulante Versorgung in Anspruch nehmen, um die Überrepräsentativität zu klären.

Schlüsselwörter

HIV · Administrative Prävalenz · Administrative Erstdiagnose · Sekundärdaten . Versorgungsforschung

\section{Prevalence of patients with HIV receiving outpatient medical care in Germany-an analysis based on secondary data}

\section{Abstract}

Background. The Robert Koch Institute (RKI) annually publishes an estimate of the number of new HIV diagnoses and the total number of people diagnosed with HIV in Germany. So far, only medication prescription data have served as secondary data as a basis for such estimates.

Objectives. In this study, we used billing data from the outpatient sector to estimate the number of patients with newly diagnosed HIV, the overall number of patients with HIV, and the HIV test rates in those with statutory health insurance in Germany.

Materials and methods. We analyzed billing data from the outpatient sector for all persons covered by statutory health insurance between 2009 and 2018. We designed annual cohorts of patient for the years 2011 to 2017 and analyzed the number of HIV diagnoses, the number of HIV-related care services, and HIV testing rates.

Results. Every year, about 6000 new patients with HIV are treated in outpatient care. The total number of patients with HIV in 2011 was about $59,300(0.106 \%)$, which increased to $80,800(0.141 \%)$ in 2017 . The average increase in the total number of patients per year of about 3600 was significantly below the estimated number of newly treated patients for each year.
Conclusion. The results may provide an indication of patients receiving HIV care in the outpatient sector. The secondary data provide the possibility of developing another epidemiological data source for population-based representation of the administrative prevalence of HIV. To clarify over-representation, there is a need for further research on patients who are using outpatient care for the first time.

Keywords

HIV - Administrative prevalence - First

diagnosis $\cdot$ Secondary data $\cdot$ Outcome research
Jahre 2011-2017 gebildet. Es wurden alle weiblichen und männlichen Patientenentitäten ab einem Alter von 15 bis 84 Jahren erfasst, die im entsprechenden Jahr frühestens im ersten Quartal und spätestens im vierten Quartal einen Test (Indexquartal) in Anspruch genommen hatten. Falls keine Testung vorlag, wurde als Indexquartal das Quartal mit der ersten im Jahr in Anspruch genommenen Leistung oder Diagnose herangezogen. Die aufgegriffenen Patientenentitäten (kurz:
Patienten) wurden insgesamt 4 Quartale (inkl. Indexquartal) nachbeobachtet.

Zur Untersuchung der erstmals ambulant versorgten Patienten mit HIV ist es notwendig, Patienten mit einer vorher bestehenden HIV-Infektion auszuschließen (Studienkohorte). Somit durfte für diese Patienten in 8 Vorquartalen vor dem Indexquartal keine gesicherte Diagnose und/oder HIV-Betreuungsleistung (M2Q) abgerechnet worden sein. Patientenentitäten, welche bereits mit einer erstmals ambulant versorgten HIV-
Infektion in eine Kohorte eingegangen waren, wurden als prävalent in folgenden Jahreskohorten geführt.

Um die Inanspruchnahme von HIVTests zu untersuchen, wurde im Beobachtungszeitraum betrachtet, ob mindestens einmal eine relevante GOP abgerechnet wurde (•Tab. 1). Zur Ermittlung der Erkrankungsraten wurde im gleichen Beobachtungszeitraum untersucht, ob bei den Patienten mindestens zweimal in unterschiedlichen Quartalen (M2Q) eine der relevanten gesicherten Diagnosen ge- 
mäß ICD-10 codiert (im Folgenden „Diagnosen“ genannt) oder eine HIV-Betreuungsleistung abgerechnet wurde $[17,18]$.

\section{Statistische Analysen}

Für die Berechnung der Anzahl erstmals ambulant versorgter Patienten mit HIV und der Testraten stellt die Grundgesamtheit die jeweilige Studienkohorte dar. Die Bestimmung der administrativen Prävalenz (Anzahl ambulant versorgter Patienten mit HIV) bezieht sich auf die Patientenkohorte. Es wurde jeweils der Anteil an Patienten im Verhältnis zur Gesamtzahl der Patienten-/Studienkohorte abgebildet.

Im Exkurs werden die Berechnungen auf Basis von ambulanten Abrechnungsdaten den Ergebnissen des RKI zu HIV gegenübergestellt. Die Daten des RKI wurden mittels der amtlichen Meldungen zu den gesetzlich Krankenversicherten (KM-6-Statistik) auf Basis der Altersgruppen auf das Niveau gesetzlich Versicherter hochgerechnet [20].

\section{Ergebnisse}

\section{Studienkohorten}

- Abb. 1 stellt exemplarisch für das Jahr 2011 das Flussdiagramm zur Bildung der Patienten- und Studienkohorte dar. Darin werden schematisch die Bildung der Studienkohorten sowie das detaillierte Vorgehen der Abgrenzung aus methodischer Sicht aufgezeigt. - Tab. 2 zeigt alle Patienten- und Studienkohorten von 2011 bis 2017.

Durchschnittlich $90 \%$ der rund 72 Mio. Versicherten nehmen mindestens eine ambulante Leistung in den Untersuchungszeiträumen in Anspruch [21-23]. Das Durchschnittsalter der Patienten in der Patientenkohorte betrug bei der Inanspruchnahme der Versorgung (Alter im Indexquartal des jeweiligen Jahres) 49 Jahre mit einer Standardabweichung von 19 Jahren. Die Hälfte der Patienten war bei der Inanspruchnahme der Versorgung im Alter zwischen 33 und 64 Jahren (Median: 49 Jahre).
Ambulant versorgte Patienten mit bestehendem HIV

Im Jahr 2011 wurden 0,095\% $(n=53.130)$ und in $20170,130 \%(n=74.900)$ der Patienten der jeweiligen Patientenkohorte wegen einer gesicherten HIV-Diagnose oder durch eine vorliegende HIV-spezifische Leistung (M2Q), welche bereits in bis zu 2 Vorjahren diagnostiziert oder in einer vorherigen Kohorte als Patient mit bestehendem HIV aufgenommen worden war, aus den weiteren Analysen ausgeschlossen (- Tab. 2). Die Patienten in den verbleibenden Studienkohorten bilden somit die Grundgesamtheit für die weitere Untersuchung der erstmals ambulant versorgten Patienten mit HIV.

\section{Erstmals ambulant versorgte Patienten mit HIV}

Als erstmals ambulant versorgte Patienten mit HIV wurden durchschnittlich $0,011 \%$ der Patienten in den Studienkohorten dokumentiert. Für 0,008\% der erstmals ambulant versorgten Patienten mit einer Diagnose HIV wurde diese dokumentiert, ohne dass im Untersuchungszeitraum eine ambulante Testung abgerechnet wurde. Im Jahr 2011 entsprach dies 4483 erstmals ambulant versorgten Patienten mit HIV ohne direkt vorausgegangenen Test (2017: $n=4348)$. Bei $0,003 \%$ der erstmals ambulant versorgten Patienten wurde mindestens ein Test durchgeführt (• Tab. 1). Im Jahr 2011 entsprach dies 1685 Patienten (2017: $n=1530)$. Erstmals ambulant versorgte Patienten mit HIV (insgesamt) kommen in allen Altersgruppen vor, die Hälfte der Patienten war zwischen 31 und 50 Jahre alt (MW: 42 Jahre, Median: 40, SD: 15 Jahre). Bei differenzierter Betrachtung der Patienten mit und ohne Testung lässt sich eine deutliche Altersverschiebung erkennen. Im Durchschnitt waren Patienten mit einer erstmals ambulant versorgten HIV-Infektion und einer Testung mit 38 Jahren 5 Jahre jünger als erstmals ambulant versorgte Patienten ohne Testung im ambulanten Bereich (Median mit Test: 36 Jahre; ohne Test: 42 Jahre).

Die Anzahl der erstmals ambulant versorgten Patienten mit HIV beträgt durchschnittlich etwa 6000 und liegt damit deutlich über dem durchschnittlichen Zuwachs in den einzelnen Patientenkohorten (• Abb. 2).

\section{Administrative Prävalenz von HIV in der ambulanten Versorgung}

Betrachtet man die Kohorten unabhängig von den bereits zuvor ambulant versorgten Patienten mit HIV-Infektion, befanden sich von 56 Mio. Patienten der Patientenkohorte im Jahr 2011 insgesamt $59.298(0,106 \%)$ mit einer HIVInfektion in der ambulanten Versorgung. Bis zum Jahr 2017 konnte ein Anstieg um 0,035 Prozentpunkte auf 0,141\% verzeichnet werden $(n=80.778)$. Dies entsprach durchschnittlich pro Jahreskohorte etwa einer Steigerung um $3500 \mathrm{~Pa}$ tienten. Hier besteht eine Diskrepanz zwischen dem durchschnittlichen Anstieg der ambulant versorgten Patienten mit HIV um etwa 2500 und der Zahl jährlich erstmals ambulant versorgter Patienten mit HIV-Infektion. Im Durchschnitt waren die versorgten Männer und Frauen 47 Jahre alt (Frauen: SD: 14 Jahre; Median: 46 Jahre; Männer: SD: 12 Jahre; Median: 47 Jahre; - Abb. 3).

\section{Darstellung der Inanspruchnahme von HIV-Tests in der ambulanten Versorgung}

- Abb. 4 stellt die Inanspruchnahme von HIV-Tests in der ambulanten Versorgung dar. Diese lag zwischen 1,62\% (2011) und 1,96\% (2017) in den Jahreskohorten. Im Jahr 2011 nahmen rund 910.000 Patienten Antikörpersuchtests in Anspruch, die Zahl erhöhte sich im Jahr 2017 um knapp 215.000 auf rund 1,12 Mio. Suchtests (+23,7\%). Knapp 2 Drittel der Patienten mit einem Suchtest erhielten diesen im Rahmen der Mutterschaftsvorsorge (2011: ca. 556.500, 2017: ca. 706.000). Nahmen 2011 noch lediglich rund 175.000 Männer einen Antikörpersuchtest in Anspruch, waren es 2017 bereits rund 217.000 Männer $(+23,6 \%)$. Einen Absicherungstest erhielten in den einzelnen Kohorten jährlich durchschnittlich 2500 Patienten (2011: 50,7\% Männer, 49,3\% Frauen; 2017: 44,4\% Männer, 55,6\% Frauen). 


\section{Originalien und Übersichten}

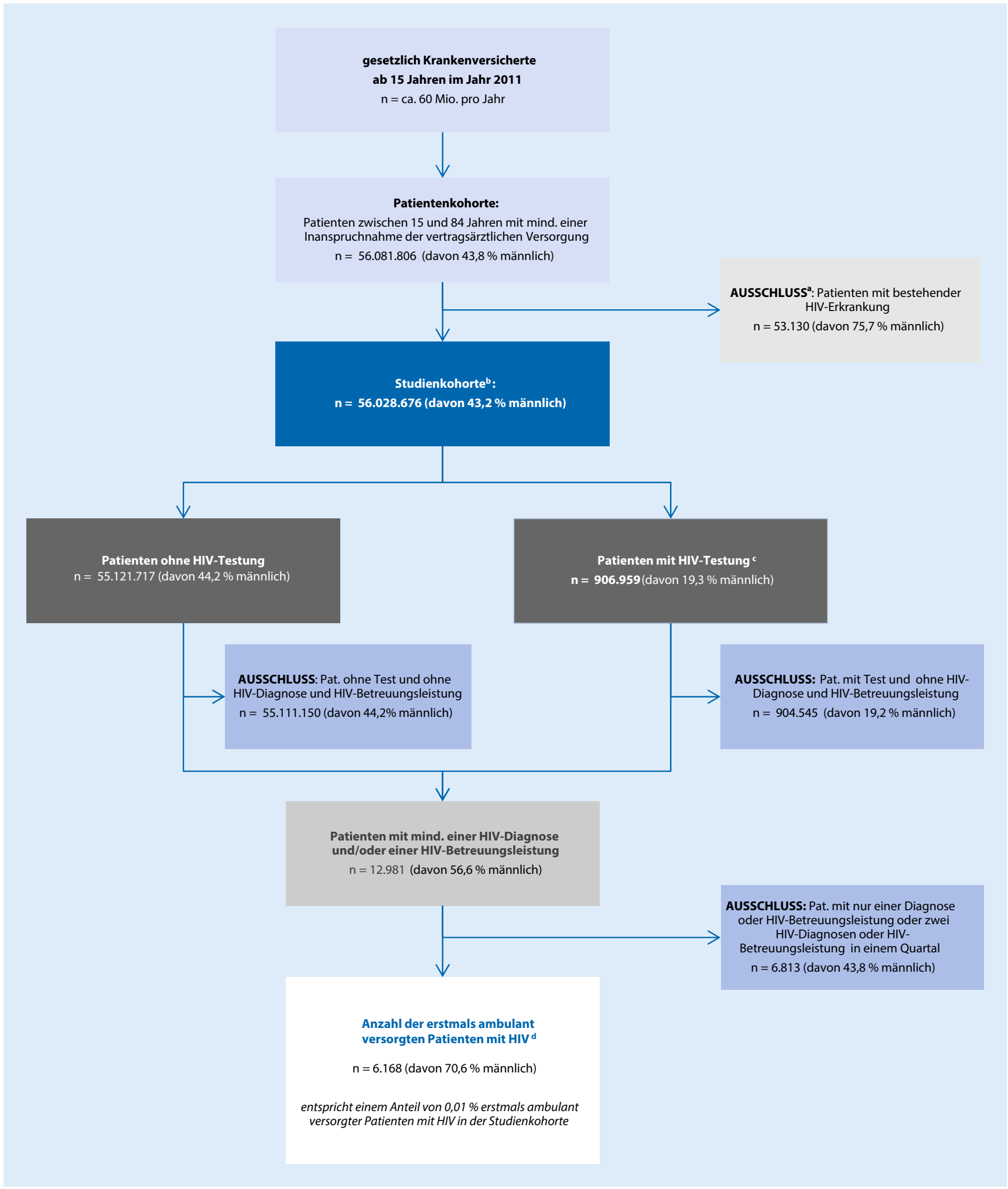

Abb. 1 A Flussdiagramm zur Bildung der Patienten- und Studienkohorte. Patienten, bei denen in den 2 Vorjahren vor dem Kohortenjahr in mindestens 2 Quartalen entweder eine HIV-Diagnose oder eine HIV-Betreuungsleistung codiert bzw. abgerechnet wurde oder die in einer vorhergehenden Kohorte mit einer HIV-Erkrankung geführt wurden, ${ }^{b}$ Patienten in der ambulanten Versorgung ohne bestehenden HIV, Patienten mit mindestens einer Leistung gemäß dem Operationalisierungskriterium Test (•Tab. 1), dbei denen in mindestens 2 Quartalen entweder eine HIV-Diagnose oder eine HIV-Betreuungsleistung codiert bzw. abgerechnet wurde, unabhängig von einer ambulanten Testung 


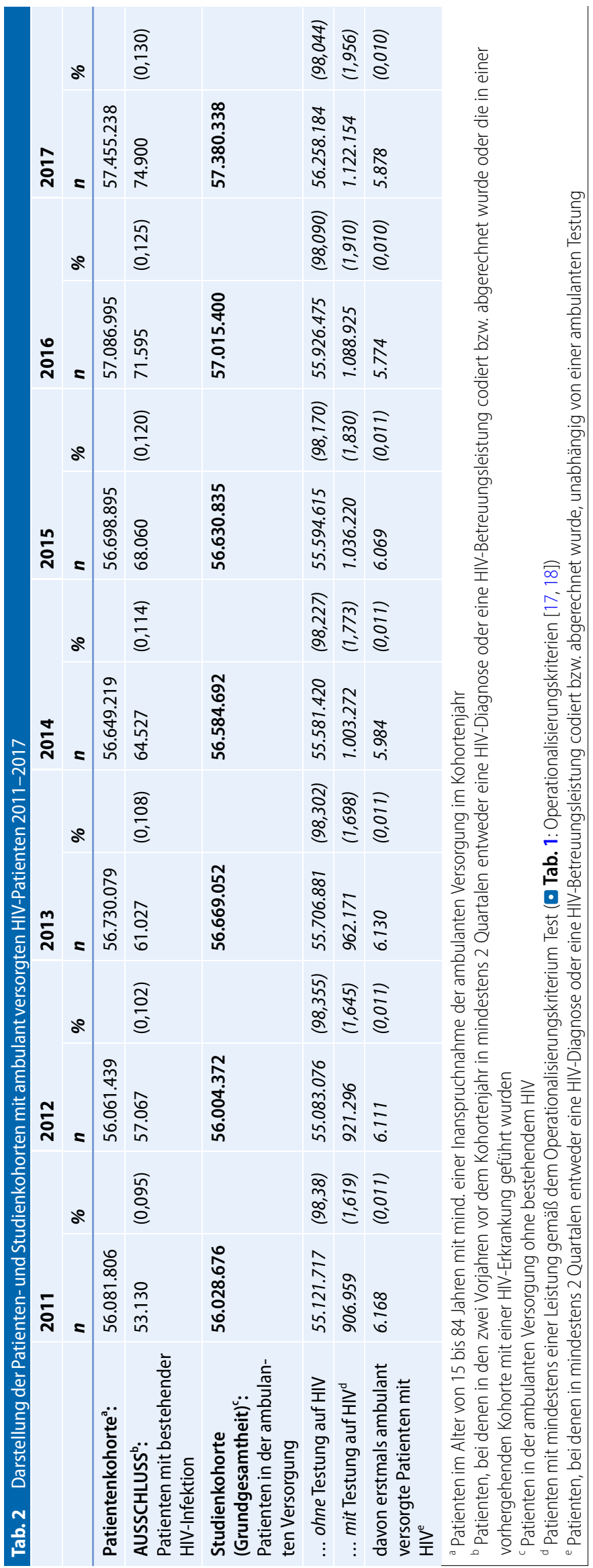

Das Durchschnittsalter der Patienten mit einer Testung (ohne Berücksichtigung der Testungen nach Mutterschaftsrichtlinie) betrug 44 Jahre (SD: 14 Jahre, Median: 36 Jahre). Die Hälfte der Patienten war zwischen 31 und 55 Jahre alt.

\section{EXKURS: Ambulante Versorgungs- daten vs. Hochrechnung auf GKV- Versicherte des RKI}

Hinsichtlich einer vergleichenden Betrachtung der ambulanten Versorgungsdaten und der Hochrechnungen auf GKV-Versicherte müssen diverse Einschränkungen berücksichtigt werden, welche sich aus der Methodik, den Eigenschaften und der primären $\mathrm{Be}$ stimmung der Daten (Sekundärdaten vs. Hochrechnung) ergeben. Die methodischen Aspekte werden ausführlich in der Diskussion dargestellt. In den Kohorten zeigen sich hinsichtlich der Anzahl der erstmals ambulant versorgten Patienten mit HIV (insgesamt) als auch in den RKI-Daten zu HIV-Erstdiagnosen annähernd gleiche Trends. Die Anzahl der erstmals ambulant versorgten Patienten mit mindestens einer ambulanten Testung und HIV blieb über die Kohorten konsistent (-Abb. 2). Betrachten wir über die Zeiträume die Streuung in den jeweiligen Altersgruppen der erstmals ambulant versorgten Patienten mit HIV und der HIV-Erstdiagnosen, zeichnet sich außer bei der Altersgruppe 70+ ein ähnlicher Trend ab [4].

Die Entwicklung der Anzahl von Patienten mit HIV in der ambulanten Versorgung zwischen 2011 und 2017 zeigt den gleichen zunehmenden Verlauf wie die Anzahl GKV-Versicherter mit HIV-Diagnose, die das RKI berechnet hat (- Abb. 3). Die ambulanten Versorgungszahlen liegen über den Hochrechnungen des RKI (Vergleich Punktschätzer: 2011: +30,1\%, 2017: +28,1\%; oberer Schätzer: 2011: $+16,7 \%, 2017:+16,3 \%$; unterer Schätzer: 2011: $+47,0 \%, 2017:+41,6 \%)$. Die Streuung in den jeweiligen Altersgruppen weist nach Geschlecht und Altersgruppen durchaus Unterschiede auf [4]. 


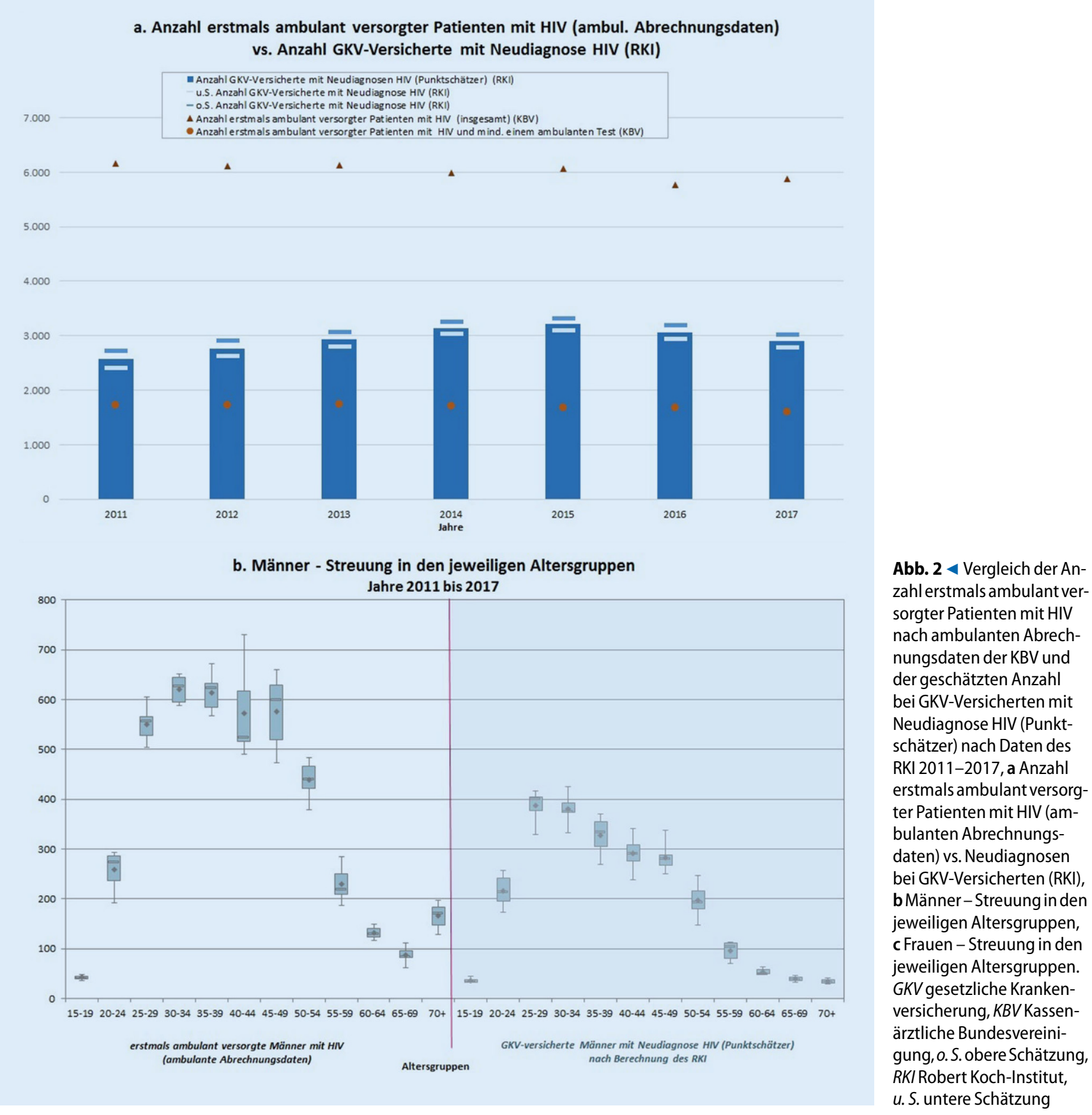

\section{Diskussion und Zusammen- fassung}

Die vorliegende Untersuchung präsentiert erstmals deutschlandweite Ergebnisse auf Basis von Sekundärdaten in Form von Leistungsdaten $\mathrm{zu}$ der Anzahl von erstmals ambulant versorgten Patienten mit HIV, der administrativen Prävalenz von HIV sowie zur Anzahl von durchgeführten Testungen zur HIV-Dia- gnostik, bei allen gesetzlich Krankenversicherten in der ambulanten Versorgung.

\section{Erstmals ambulant versorgte Patienten mit HIV und Testraten}

Der in der Untersuchung dargestellte Anteil der erstmals ambulant versorgten Patienten mit HIV lag in den Jahren 2011 bis 2017 bei $0,011 \%$. Diese Zahl liegt in allen Jahren deutlich über den Hochrechnungen des RKI zu GKV-Versicherten mit HIV-Erstdiagnosen. Insgesamt bleibt der Anteil von erstmals ambulant versorgten Patienten mit HIV über die Jahre stabil (•Abb. 3). Die Ergebnisse unterstreichen die Schwierigkeiten bei der Bestimmung der HIV-Erstdiagnosen in Deutschland. Den Hochrechnungen auf GKV-Versicherte mit einer HIV-Erstdiagnose liegen Schätzungen des RKI zugrunde, welche auf verschiedenen Datenquellen (z.B. Aidsfallregister, IfSGMeldedaten, Todesursachen) basieren. 


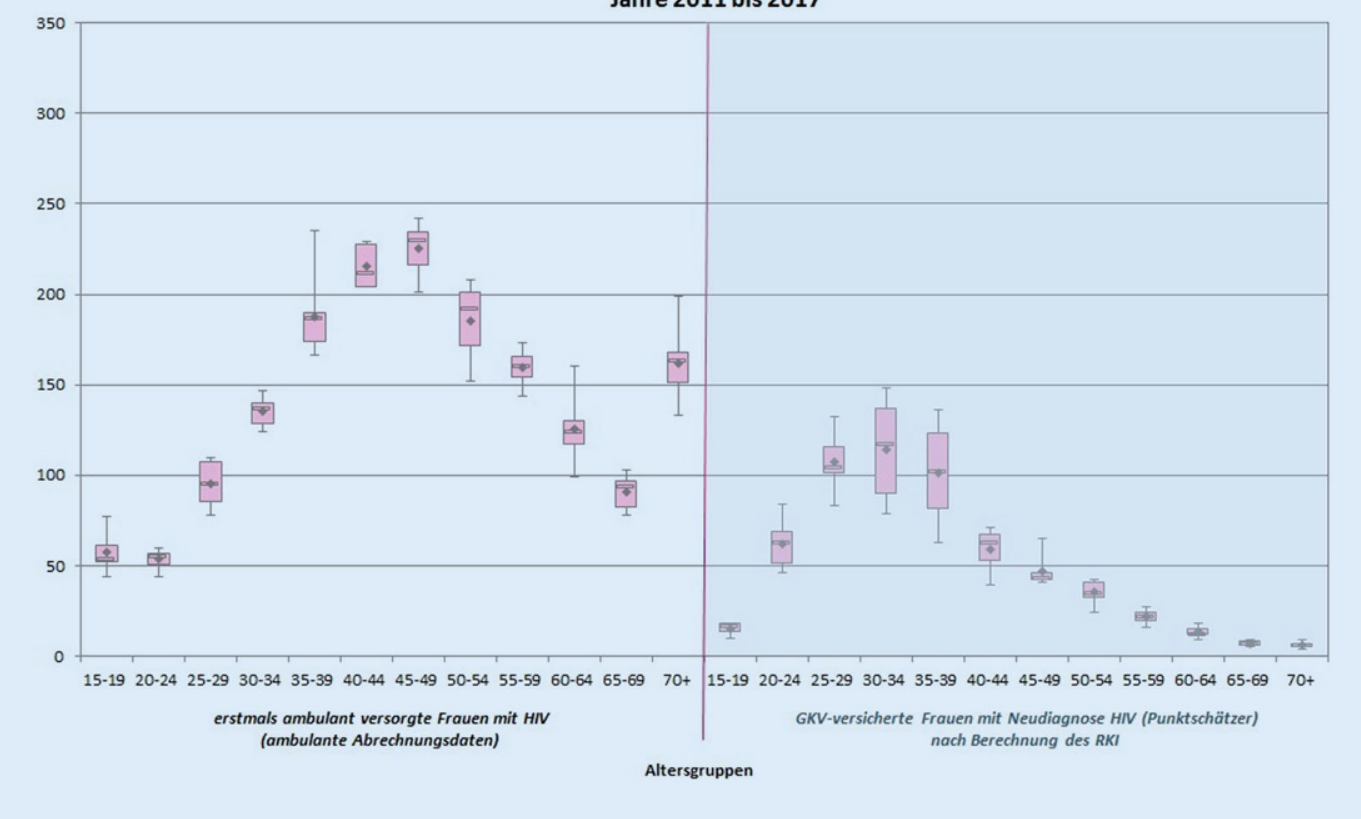

Abb. $2 \triangleleft$ (Fortsetzung)

Die Meldungen hierzu enthalten sowohl die gemeldeten Fälle aus der ambulanten Versorgung als auch die durch spezialisierte Einrichtungen festgestellten Fälle (z.B. Gesundheitsamt, Deutsche AIDSHilfe, stationäre Einrichtungen) sowie Todesfälle. Der Unterschied zwischen den Ergebnissen lässt vermuten, dass es häufiger zu einer zeitlichen Verzögerung zwischen der Testung und der ersten ambulanten Versorgung kommt. Der hohe Anteil an Patienten mit einem erstmals ambulant versorgten HIV ohne Testung im ambulanten Bereich kann einen Hinweis darauf geben, dass Patienten nach einer bekannt gewordenen HIVDiagnose zunächst keine weiterführende Behandlung in Anspruch nehmen. Dies deutet darauf hin, dass die im Jahr 2014 eingeführte Leitlinienempfehlung, möglichst direkt nach der HIVDiagnose eine antiretrovirale Therapie zu beginnen, noch nicht flächendeckend umgesetzt wird. Ein früher Therapiestart kann die negativen Auswirkungen einer chronischen HIV-Infektion reduzieren, darüber hinaus vermindert eine erfolgreiche Therapie die Weitergabe von HIV [24]. Weiterhin könnte als Grund der hohen Zahl ein Wechsel aus der spezialisierten Versorgung in die ambulante
Versorgung hinein in Betracht gezogen werden.

\section{Administrative Prävalenz in der ambulanten Versorgung}

Die vorliegende administrative Prävalenz von ambulant versorgten Patienten mit HIV lag im Jahr 2011 bei 0,106\% $(n=59.289)$ und stieg kontinuierlich bis zum Jahr 2016 um 0,035 Prozentpunkte $(n=80.778)$ an. Die sich damit ergebende Diskrepanz zwischen dem durchschnittlichen Anstieg dieser Gesamtzahl um etwa 2500 und der geschätzten Zahl jährlich erstmals ambulant versorgter Patienten kann zum Teil mit spezialisierter Inanspruchnahme von Versorgung (z.B.Hochschulambulanzen) oder einem Versterben der Patienten erklärt werden. Eine mögliche Überschätzung lässt sich jedoch nicht völlig ausschließen. In den Jahreskohorten lag die administrative Prävalenz in der ambulanten Versorgung durchschnittlich leicht über den Hochrechnungen auf GKV-Versicherte mit HIV/Aids in Deutschland. Die Hochrechnung auf GKV-Versicherte des RKI könnte die tatsächliche Prävalenz unterschätzen, da beispielsweise nach einer bekannt gewordenen HIV-Infektion bei Privatversicherten ein Wechsel in die gesetzliche Krankenversicherung aufgrund der Kosten in Betracht gezogen werden kann. Hinzu kommen gegebenenfalls Fehler oder Verzögerungen bei den Meldungen nach IfSG, welche in die Hochrechnungen auf GKV-Versicherte mit HIV/Aids in Deutschland eingegangen sein könnten.

\section{Stärken und Limitationen}

Die Datenbasis der vorliegenden Studie entspricht der Population aller gesetzlich krankenversicherten Personen, die die ambulante kollektivvertragliche Versorgung in Anspruch nehmen, und bildet somit die krankenkassen- und regionsübergreifende Versorgungsrealität hinsichtlich des Leistungs- und Codiergeschehens ab.

Bisherige Untersuchungen der administrativen Prävalenz oder Erstdiagnosen von HIV in Deutschland waren auf bestimmte Krankenkassen oder Teilpopulationen beschränkt und haben eine entsprechend eingeschränkte Repräsentativität für die Gesamtpopulation [2-4, 13, 23, 25-27].

Die vorliegenden Analysen beziehen sich auf mehrere Jahre und liefern somit einen ersten Einblick in die Entwicklung der administrativen Prävalenz von HIV, 


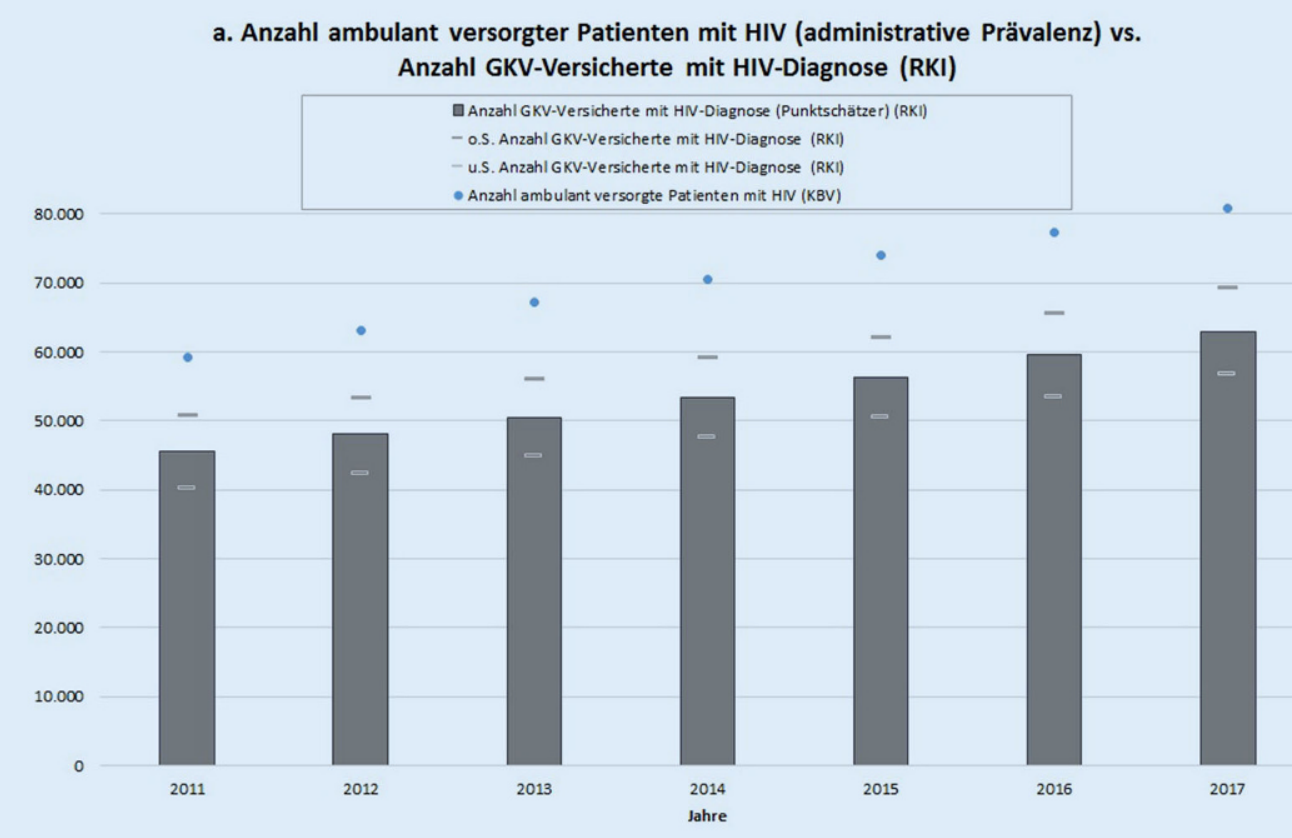

b. Männer - Streuung in den jeweiligen Altersgruppen Jahre 2011 bis 2017

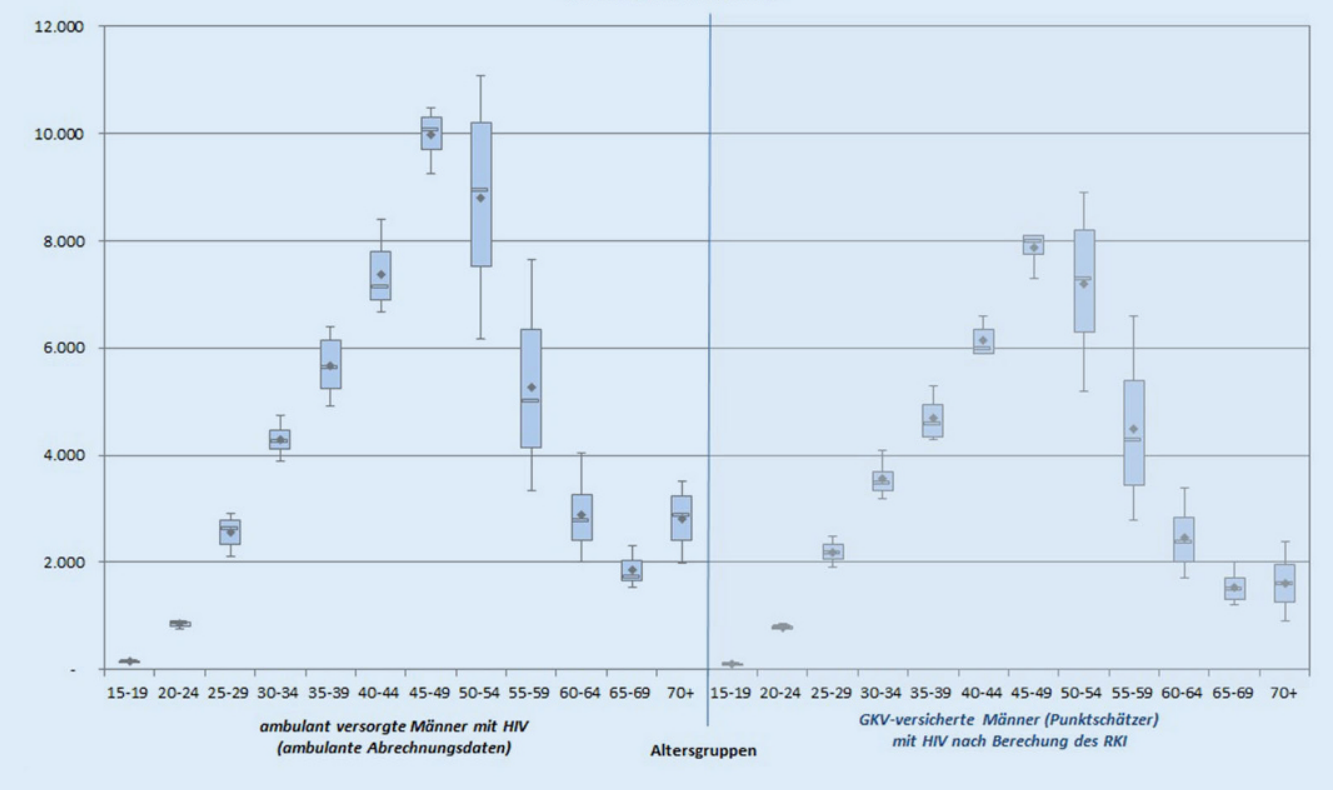

Abb. $3<$ Vergleich der Anzahl von ambulant versorgten Patienten mit HIV (administrative Prävalenz) nach ambulanten Abrechnungsdaten der KBV und der geschätzten Anzahl bei GKV-Versicherten mit HIV-Diagnose (Punktschätzer) nach Daten des RKI 2011-2017. a Anzahl ambulant versorgter Patienten mit HIV (administrative Prävalenz) vs. Anzahl der GKV-Versicherten mit HIVDiagnose (Punktschätzer), b Männer-Streuung in den jeweiligen Altersgruppen, c Frauen - Streuung in den jeweiligen Altersgruppen. GKV gesetzliche Krankenversicherung, KBV Kassenärztliche Bundesvereinigung, o. S. obere Schätzung, RKI Robert Koch-Institut, u. S. untere Schätzung der erstmals ambulant versorgten Patienten mit HIV und der Testungen auf Basis von Sekundärdaten für Deutschland.

Eine Einschränkung der Studie liegt in der begrenzten Aussagefähigkeit von Sekundärdaten (administrativen Daten), die nicht für Forschungszwecke erfasst werden und mehrere Schwächen aufweisen. Diagnosen, welche ohne entsprechende Testung auftreten, können möglicherweise im stationären Setting gestellt worden sein oder aus anderweitig wahrgenommenen Testangeboten (z.B. Aidshilfe, Gesundheitsamt), bei denen HIV diagnostiziert wurde, übertragen worden sein. Schwierig gestaltet sich die Bestimmung der Prävalenz und Inzidenz von HIV aufgrund der schwer bestimmbaren Latenzzeit zwischen dem Zeitpunkt der Infektion und dem Zeitpunkt der Ersterfassung als HIV-Fall im Gesundheitssystem beim RKI oder in der ambulanten Versorgung. Vor diesem Hintergrund muss beachtet werden, dass die Erfassung eines „erstmals ambulant versorgten Patienten“ und den vom RKI dargestellten „Erstdiagnosen GKV-Versicherte" nicht gleichzeitig erfolgt, da Patienten die ambulante Versorgung zu einem späteren Zeitpunkt erstmalig in Anspruch nehmen können, ein Wechsel von der privaten zur gesetzlichen Krankenversicherung vorliegen könnte oder auch ggf. Meldeverzögerungen eine Rolle spielen. Die dargestellte administrative Prävalenz stellt abhängig 
c. Frauen - Streuung in den jeweiligen Altersgruppen Jahre 2011 bis 2017

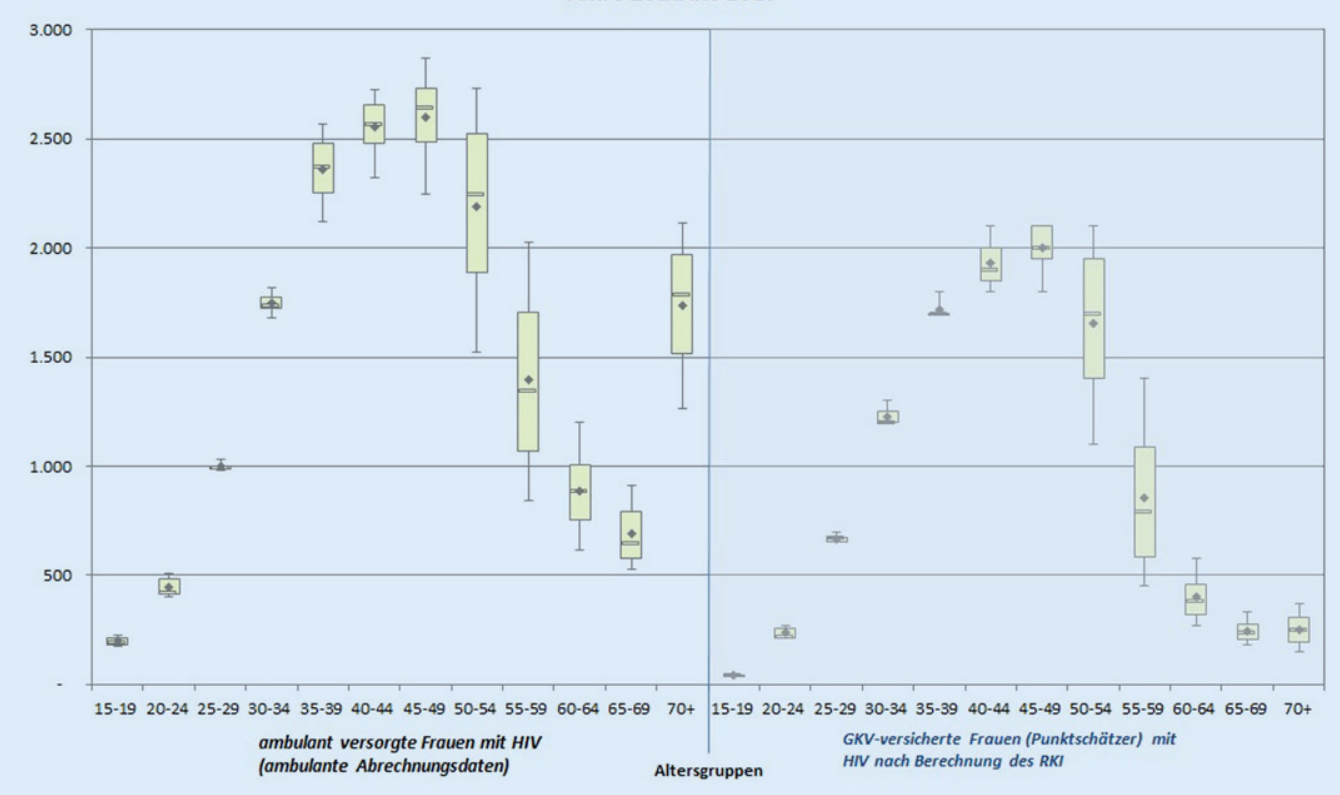

Abb. $3<$ (Fortsetzung)

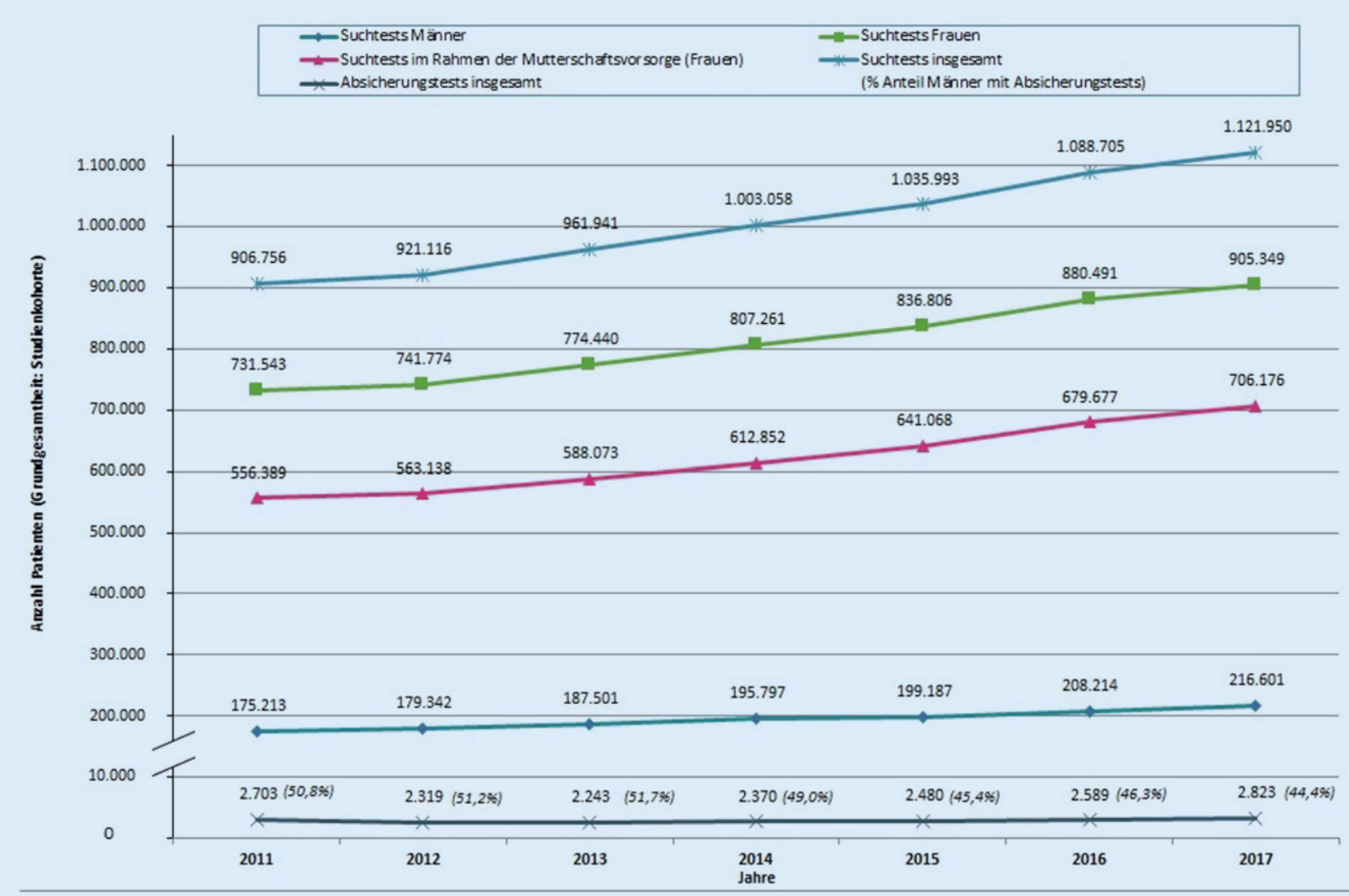

Abb. $4 \Delta$ Darstellung der Inanspruchnahme der Antikörpersuchtests und Absicherungstests in den Studienkohorten differenziert nach Geschlecht und im Rahmen der Testung (Mutterschaftsvorsorge) 
von der Datengrundlage eine gewisse Annäherung an die tatsächliche Prävalenz der gesetzlich Versicherten dar. Ein direkter jahresbezogener Vergleich der Berechnungen auf Basis von Sekundärdaten und den Hochrechnungen auf GKV-Versicherte des RKI ist zudem nur eingeschränkt möglich, aufgrund der unterschiedlichen Definition und Auswahl der untersuchten Grundgesamtheit, der Datengrundlagen, der Operationalisierung der administrativen Prävalenz bzw. erstmals ambulant versorgten Patientenentitäten mit HIV sowie des zeitlichen Verzuges. Daten des RKI berücksichtigen in den Schätzungen beispielsweise die Daten der Todesursachenstatistik. In den Daten dieser Studie kann ein Versterben der Patienten nicht berücksichtigt werden.

Für die Definition und Auswahl der untersuchten Grundgesamtheit wurde eine inhaltlich-medizinisch abgeleitete und transparent dargestellte Kohortenbildung angestrebt, um eine möglichst valide Bestimmung der Patienten mit HIV zu erreichen. Verglichen mit öffentlichen Statistiken zur Anzahl der gesetzlich Versicherten vom 15. bis zum 84. Lebensjahr ergibt sich eine Abdeckung von ca. 95\% in den Kohorten, welchegleichzeitig die Inanspruchnahme von Versicherten widerspiegelt. Mehreren nationalen Studien zufolge nehmen 90,9\% der Frauen und 84,1\% der Männer (insgesamt: 90\%) innerhalb eines Jahres ambulante ärztliche Leistungen in Anspruch [21-23]. Die Abdeckung liegt etwas über der geschätzten Inanspruchnahme von gesetzlichen Versicherten. Dies ist auf die Bildung der Patientenentitäten in den verwendeten Abrechnungsdaten zurückzuführen. Die Patientenentitäten werden über eine Kombination aus vorliegenden Pseudonymattributen (Namen, Vorname und Geburtsdatum) gebildet, sodass beispielsweise Namenswechsel oder Fehler bei der manuellen Erfassung im Rahmen des Ersatzverfahrens in Praxen zu einer Verdünnungsquote beitragen. Die Patientenzahl in den Abrechnungsdaten wird jeweils für das entsprechende Jahr ins Verhältnis gesetzt zu der Anzahl Krankenversicherter (KM-6Statistik) und dieser Quotient wird als
Verdünnungsquote bezeichnet [20, 28]. Zudem können Patientenentitäten, die im deutschen Gesundheitssystem nicht gesetzlich versichert sind, über die Leistungsdaten nicht erfasst werden.

\section{Fazit}

Die vorliegenden Sekundärdaten geben die Möglichkeit, eine weitere epidemiologische Datenquelle zur bevölkerungsbezogenen Darstellung der administrativen Prävalenz von HIV zu erschließen. Die administrative Prävalenz liegt mit 0,141\% in 2017 im Bereich der aktuellen Schätzungen des RKI. Weiterhin können durch die Datengrundlage Lücken hinsichtlich der Versorgung und Inanspruchnahme geschlossen werden. Die Ergebnisse können die kontinuierliche Berichterstattung des RKI ergänzen sowie für weiterführende mathematischdemografische Bestimmungen herangezogen werden. Es besteht weiterhin Forschungsbedarf zu Patienten, welche erstmals Versorgung im ambulanten Sektor in Anspruch nehmen, um die Überrepräsentativität zu klären.

\section{Korrespondenzadresse}

Diana Kurch-Bek, MPH, Dipl.Wirtschaftsinformatikerin (FH) Innovation, strategische Analyse und ITBeratung, Kassenärztliche Bundesvereinigung Herbert-Lewin-Platz 2, 10623 Berlin,

Deutschland

dkurch-bek@kbv.de

\section{Einhaltung ethischer Richtlinien}

Interessenkonflikt. D. Kurch-Bek, C. Gallowitz, A. Baumgarten, B. Tenckhoff und M. an der Heiden geben an, dass kein Interessenkonflikt besteht.

Für diesen Beitrag wurden von den Autoren keine Studien an Menschen oder Tieren durchgeführt. Für die aufgeführten Studien gelten die jeweils dort angegebenen ethischen Richtlinien.

Open Access. Dieser Artikel wird unter der Creative Commons Namensnennung 4.0 International Lizenz veröffentlicht, welche die Nutzung, Vervielfältigung, Bearbeitung, Verbreitung und Wiedergabe in jeglichem Medium und Format erlaubt, sofern Sie den/die ursprünglichen Autor(en) und die Quelle ordnungsgemäß nennen, einen Link zur Creative Commons Lizenz beifügen und angeben, ob Änderungen vorgenommen wurden.
Die in diesem Artikel enthaltenen Bilder und sonstiges Drittmaterial unterliegen ebenfalls der genannten Creative Commons Lizenz, sofern sich aus der Abbildungslegende nichts anderes ergibt. Sofern das betreffende Material nicht unter der genannten Creative Commons Lizenz steht und die betreffende Handlung nicht nach gesetzlichen Vorschriften erlaubt ist, ist für die oben aufgeführten Weiterverwendungen des $\mathrm{Ma}$ terials die Einwilligung des jeweiligen Rechteinhabers einzuholen.

Weitere Details zur Lizenz entnehmen Sie bitte der Lizenzinformation auf http://creativecommons.org/ licenses/by/4.0/deed.de.

\section{Literatur}

1. UNAIDSUNAIDSDATA (2017) https://www.unaids org/en/resources/documents/2017/2017_data_ book. Zugegriffen: 25. Febr. 2020

2. Robert Koch-Institut (RKI) (2017) Epidemiologisches Bulletin 47/2017. Schätzung der Zahl der HIV-Neuinfektionen und der Gesamtzahl von Menschen mit HIV in Deutschland Stand Ende 2016. https://www.rki.de/DE/Content/Infekt/ EpidBull/Archiv/2017/Ausgaben/47_17.pdf? blob=publicationFile. Zugegriffen: 25 . Febr. 2020

3. Robert Koch-Institut (RKI) (2017) Epidemiologisches Bulletin 39/2017. HIV-Jahresbericht 2016. https://www.rki.de/DE/Content/Infekt/ EpidBull/Archiv/2017/Ausgaben/39_17.pdf? blob=publicationFile. Zugegriffen: 25 . Febr. 2020

4. Robert Koch-Institut (RKI) (2018) Epidemiologisches Bulletin 47/2018. Schätzung der Zahl der HIV-Neuinfektionen und der Gesamtzahl von Menschen mit HIV in Deutschland Stand Ende 2017. https://www.rki.de/DE/Content/Infekt/ EpidBull/Archiv/2018/Ausgaben/47_18.pdf? blob=publicationFile. Zugegriffen: $2 \overline{5}$. Febr. 2020

5. European Centre for Disease Prevention and Control (ECDC) (2017) HIV testing in europe. Monitoring implementation of the Dublin declaration on partnership to fight HIV/AIDS in Europe and central Asia: 2017 progress report. https:// ecdc.europa.eu/en/publications-data/thematicreport-hiv-testing. Zugegriffen:6. Nov. 2017

6. Deutsches Ärzteblatt Paul-Ehrlich-Institut und Bundesgesundheitsministerium informieren über HIV-Selbsttest. https://www.aerzteblatt. de/nachrichten/98198/Paul-Ehrlich-Institutund-Bun-des-ge-sund-heits-mi-nis-ter-iuminformieren-ueber-HIV-Selbsttest. Zugegriffen: 25. Februar 2020

7. Deutsche AIDS-Hilfe HIV-Selbsttest. https:// www.aidshilfe.de/hiv-selbsttest. Zugegriffen: 25. Februar 2020

8. Gourlay AJ, Pharris AM, Noori T et al (2017) Towards standardized definitions for monitoring the continuum of HIV care in Europe. AIDS 31:2053-2058. https://doi.org/10.1097/QAD. 0000000000001597

9. European Centre for Disease Prevention and Control (ECDC) (2020) HIV modelling tool. https:// www.ecdc.europa.eu/en/publications-data/hivmodelling-tool.Zugegriffen: 17. März 2020

10. van Sighem A, Nakagawa F, de Angelis D et al (2015) Estimating HIV incidence, time to diagnosis, and the undiagnosed HIV epidemic using routine surveillance data. Epidemiology 26:653-660. https://doi.org/10.1097/EDE.0000000000000324

11. Nacher M, Adriouch L, Huber F et al (2018) Modeling of the HIV epidemic and continuum 
of care in French Guiana. PLoS ONE 13:e197990. https://doi.org/10.1371/journal.pone.0197990

12. Supervie V, Ndawinz JDA, Lodi S, Costagliola D (2014) The undiagnosed HIV epidemic in France and its implications for HIV screening strategies. AIDS 28:1797-1804. https://doi.org/10.1097/ QAD. 0000000000000270

13. Finkenstädt $V$, Wild $F$ HIV-Infektion in der PKV. Prävalenz, Inzidenz und Arzneimittelversorgung. http://www.wip-pkv.de/fileadmin/DATEN/ Veroeffentlichungen/HIV-Infektion_in_der_PKV. pdf.Zugegriffen: 25. Febr. 2020

14. Pomorin N, Wasem J (2008) Analyse der aktuellen HIV-Zahlen. Diskussionsbeitrag. https://www. wiwi.uni-due.de/fileadmin/fileupload/WIWI/ Forschung/IBES_Diskussionbeitraege/169.pdf. Zugegriffen:25. Febr. 2020

15. Tomeczkowski J, Mahlich J, Stoll M (2015) Fehlkodierungen von HIV/AIDS-Diagnosen und deren Bedeutung für den morbiditätsorientierten Risikostrukturausgleich (Incorrect Coding of HIV/AIDS-Diagnoses and their Relevance for Financing Statutory Sickness Funds in Germany). Gesundheitsökonomie \& Qualitätsmanagement 20(03):132-139. https://doi.org/10.1055/s-00341385779

16. Swart E, Ihle P, Gothe H, Matusiewicz D (2014) Routinedaten im Gesundheitswesen. Handbuch Sekundärdatenanalyse: Grundlagen, Methoden, und Perspektiven, 2. Aufl. Huber, Verlag Hans Huber, Bern

17. Kassenärztliche Bundesvereinigung Einheitlicher Bewertungsmaßstab (EBM) (2020) KassenärztlicheBundesvereinigung Einheitlicher Bewertungsmaßstab (EBM). https://www.kbv.de/html/ebm. php.Zugegriffen:25. Febr. 2020

18. Deutsches Institut für Medizinische Dokumentation und Information (DIMDI) (2017) Internationale statistische Klassifikation der Krankheiten und verwandter Gesundheitsprobleme. ICD-10-GM. https://www.dimdi.de/static/de/klassi/icd-10gm/kodesuche/onlinefassungen/htmlgm2017/ index.htm. Zugegriffen: 25. Febr. 2020

19. Bundesversicherungsamt (2018) Festlegung der Morbiditätsgruppen, des Zuordnungsalgorithmus, des Regressions- sowie des Berechnungsverfahrens. https://www.bundesversicherungsamt. de/risikostrukturausgleich/festlegungen.html\# c5594.Zugegriffen: 12. Sept. 2018

20. Bundesministerium für Gesundheit (2020) KM 6-Statistik. gesetzliche Krankenversicherung: Versicherte. https://www.bundesgesundheitsminis terium.de/themen/krankenversicherung/zahlenund-fakten-zur-krankenversicherung/mitgliederund-versicherte.html.Zugegriffen: 11. Febr. 2020

21. Grobe TG, Steinmann S, Szecsenyi J BARMER GEK Arztreport 2016. https://www.barmer.de/blob/ 36738/41528a9e5704bb8d47e25e00707af4ba/ data/pdf-arztreport-2016.pdf. Zugegriffen: 25 . Febr. 2020

22. Grobe TG, Steinmann S, Szecsenyi J (2017) Arztreport 2017. https://www.barmer.de/blob/99196/ 40985c83a99926e5c12eecae0a50e0ee/data/dlbarmer-arztreport-2017.pdf. Zugegriffen: 25. Febr. 2020

23. Robert Koch-Institut (2017) Inanspruchnahme ambulanter ärztlicher Versorgung in Deutschland. https://www.rki. de/DE/Content/Gesundheitsmonitoring/ Gesundheitsberichterstattung/GBEDownloadsJ/ FactSheets/JoHM_04_2017_Ambulante_ aerztliche_Versorgung.pdf?_blob=publication File.Zugegriffen:25. Febr. 2020
24. Deutsche AIDS-Gesellschaft (2014) DeutschÖsterreichische Leitlinien zur antiretroviralen Therapie der HIV-Infektion. https://www. awmf.org/uploads/tx_szleitlinien/055-001__ Antiretrovirale_Therapie_der_HIV_Infektion 2014-05.pdf.Zugegriffen: 25. Febr. 2020

25. Robert Koch-Institut (RKI) (2010) Epidemiologisches Bulletin 46/2010. Zum Verlauf der HIV-Epidemie in Deutschland bis Ende 2010. https://www. rki.de/DE/Content/Infekt/EpidBull/Archiv/2010/ Ausgaben/46_10.pdf?_blob=publicationFile. Zugegriffen:25. Febr. 2020

26. Tomeczkowski J, Mahlich J, Stoll M (2015) Häufigkeiten antiretroviraler Therapie in Sekundärdaten von gesetzlichen Krankenkassen in Deutschland (Antiretroviral therapy for HIV/AIDS in claims data from statutory health insurancefunds in Germany). Zeitschriftfür Evidenz, Fortbildung und Qualitätim Gesundheitswesen 109:594-604. https://doi.org/ 10.1016/j.zefq.2014.09.002

27. Pauly F, Freese A-L, Golic M, Henrich W, Weizsaecker K (Hrsg) (2013) Testing for HIV during pregnancy. 5 years after changing German pregnancy guidelines Bd.288. https://doi.org/10.1007/ s00404-013-2744-9

28. Mangiapane S, Riens B, Augustin J (2011) Populationsbildung auf Grundlage von Abrechnungsdaten der vertragsärztlichen Versorgung. https://www.versorgungsatlas.de/fileadmin/pdf/ Verduennungsquote_Bericht.pdf. Zugegriffen: 11. Febr. 2020 